\title{
A Case of Adenocarcinoma of the Retinal Pigment Epithelium: An Immunohistochemical and Electron Microscopic Study
}

\author{
Hidetsugu Mori Kanji Takahashi \\ Department of Ophthalmology, Kansai Medical University, Osaka, Japan
}

\section{Established Facts}

- Retinal pigment epithelium (RPE) adenocarcinoma is a very rare malignant intraocular tumor.

\section{Novel Insights}

- We describe the detailed histopathological findings of RPE adenocarcinoma by light microscopy, including immunohistochemistry and electron microscopy.

\section{Keywords}

Retinal pigment epithelium adenocarcinoma .

Malignant melanoma of the choroid - Light microscopy . Immunohistochemistry · Electron microscopy · Nevoid cell

\begin{abstract}
Purpose: Retinal pigment epithelium (RPE) adenocarcinoma is a very rare malignant intraocular tumor. Herein we describe the histopathological features of RPE adenocarcinoma. Case: A 36-year-old male was referred to our clinic because of floaters in his left eye. The initial diagnosis was malignant melanoma of the choroid. We resected the tumor and studied it histopathologically. The tumor tissue was investigated by light microscopy including immunohistochemistry using antibodies against S-100, HMB-45, EMA, and $A E-1$. Electron microscopic examination was also performed. Results: The tumor arose from the RPE and contained intracytoplasmic vacuoles and abundant melanin pigment. There
\end{abstract}

\begin{tabular}{ll}
\hline KARGER & $\begin{array}{l}\text { (c) } 2017 \text { The Author(s) } \\
\text { Published by S. Karger AG, Basel }\end{array}$ \\
$\begin{array}{l}\text { E-Mail karger@karger.com } \\
\text { www.karger.com/oop }\end{array}$ & $\begin{array}{l}\text { This article is licensed under the Creative Commons Attribution- } \\
\text { NonCommercial-NoDerivatives } 4.0 \text { International License (CC BY- } \\
\text { NC-ND) (http://www.karger.com/Services/OpenAccessLicense). } \\
\text { Usage and distribution for commercial purposes as well as any dis- } \\
\text { tribution of modified material requires written permission. }\end{array}$
\end{tabular}

were no nevoid cells in the choroid. A small part of the tumor cells showed tubular or lobular proliferation and choroidal invasion. Immunohistochemistry revealed positive staining in tumor cells with 4 antibodies. Tight cellular junctions specific to the RPE were confirmed by electron microscopy. The final diagnosis was RPE adenocarcinoma. Conclusions: Most pigmented intraocular tumors are nevus and malignant melanomas of the choroid. It is easy to misdiagnose a RPE adenocarcinoma as a malignant melanoma of the choroid. An exact differential diagnosis should be determined by immunohistopathological and electron microscopic examination.

(c) 2017 The Author(s) Published by S. Karger AG, Basel

\section{Introduction}

Adenocarcinoma of the retinal pigment epithelium (RPE) is extremely rare and difficult to diagnose clinically [1-3]. Moreover, RPE adenocarcinoma usually 

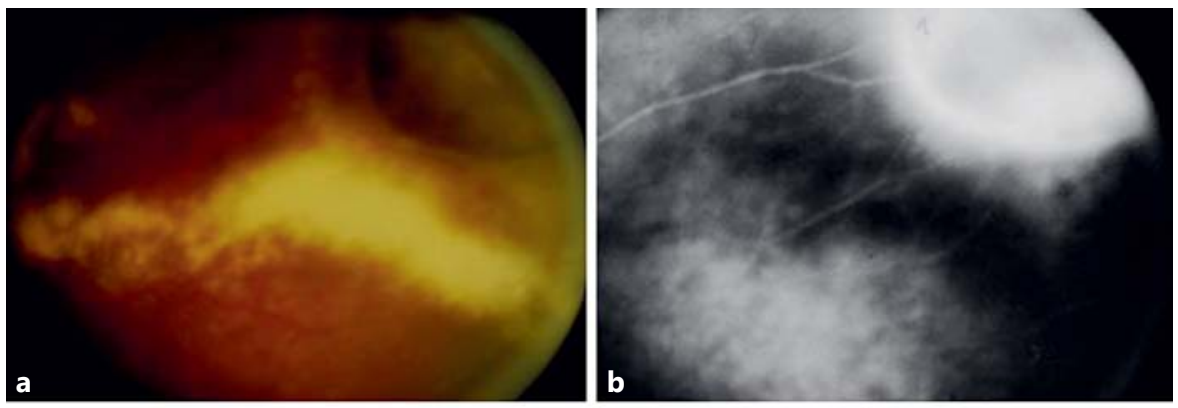

Fig. 1. Clinical findings. a Preoperative findings. Yellow exudation and a flat retinal detachment around the pigmented mass. b Fluorescein angiographic findings (late phase). The tumor is fed by retinal vessels, and extensive fluorescein leakage can be seen. c Echographic findings. The tumor has acoustic solidity and a pedunculated configuration. d, e Magnetic resonance imaging findings (d T1-weighted image; e T2-weighted image). The tumor shows a hyperintense signal in T1 imaging and a hypointense signal in T2 imaging. f Postoperative findings. Retinochoroidal defect and bare sclera are seen at the site of the resected region.
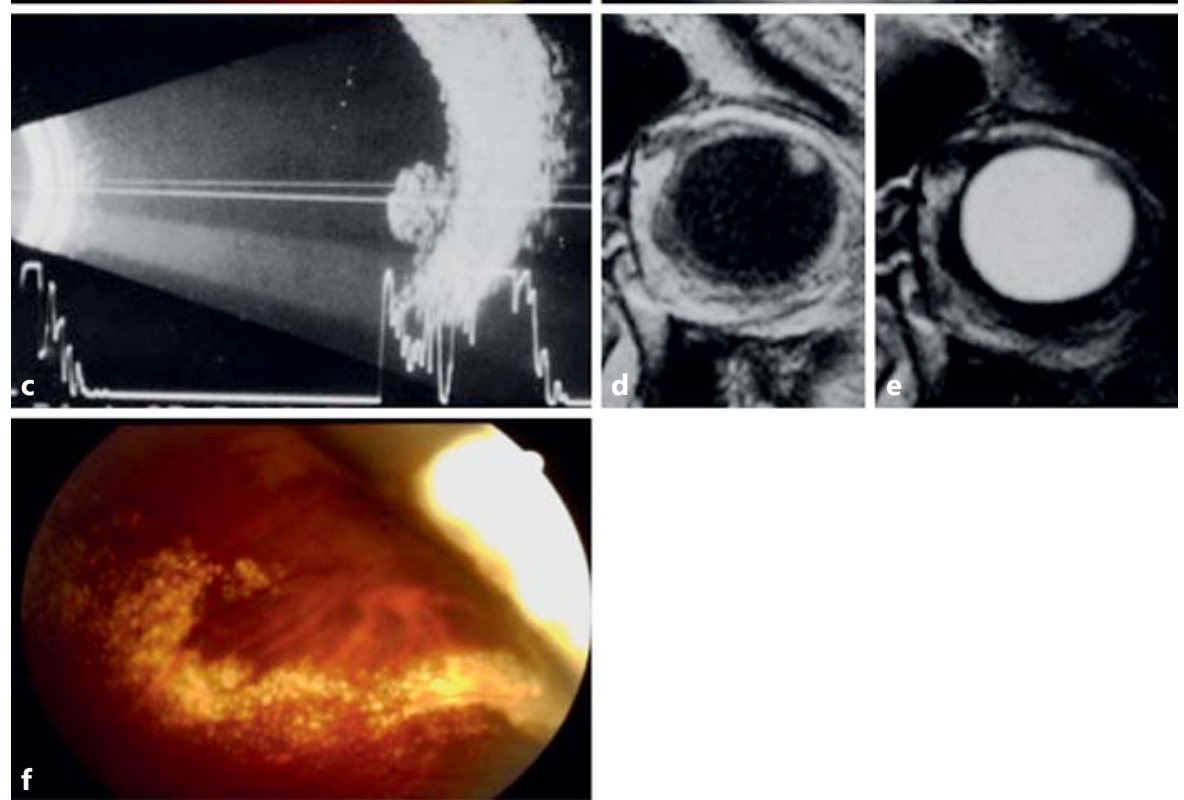

presents to the ophthalmic pathologist with a presumptive diagnosis of malignant melanoma of the choroid [2, $4,5]$. We report a histopathologically confirmed case of RPE adenocarcinoma that was clinically diagnosed as a malignant melanoma of the choroid.

\section{Case Report}

A 36-year-old male was referred to Kansai Medical University Hospital on July 7, 1994, because of floaters in his left eye for the previous 2 months. A subretinal pigmented mass was detected by the referring physician. Our initial examination revealed best-corrected distance visual acuity as 20/20 in each eye. The right eye was unremarkable. Intraocular pressure and anterior segment slitlamp examination of the left eye was normal, but there was mild vitreous opacity. Fundus examination disclosed a darkly pigmented mass at the equator superotemporally in the left eye measuring approximately 5-6 disc diameters. The mass had considerable yellowish intraretinal exudation and was surrounded by flat exudative retinal detachment (Fig. 1a). Fluorescein angiography showed early hypofluorescence and late intense leakage. Moreover, fluo- rescein angiography revealed a vascularized tumor with some of the blood supply appearing from the retinal vasculature (Fig. 1b). B-scan ultrasonography demonstrated a pedunculated mass with acoustic solidity and no choroidal excavation (Fig. 1c). Magnetic resonance imaging findings showed a hyperintense focus on T1weighted images and a hypointense focus on T2-weighted images (Fig. 1d, e). The clinical diagnosis was malignant melanoma of the choroid [5]. The mass was totally resected with a transscleral approach with pars plana vitrectomy and an encircling procedure on August 31, 1994. This operation was performed without complications [5]. Postoperative fundus examination showed retinochoroidal defect and bare sclera at the site of the resected region (Fig. 1f). Fifty-four months following the surgery, the patient had no local recurrence and no systemic metastasis. Final visual acuity in his left eye was 20/16. Histopathological results were performed as below.

Gross inspection of the sectioned tumor disclosed a domeshaped, pigmented mass measuring $7.5 \times 5.5 \mathrm{~mm}$ in basal diameter and $3.5 \mathrm{~mm}$ in thickness that was located on the choroid in the superotemporal region. The tumor arising from the ora serrata was raised highly toward the retina, partially including the pars plana (Fig. 2a).

Microscopic examination of hematoxylin and eosin-stained samples showed that the tumor was abruptly elevated from the 

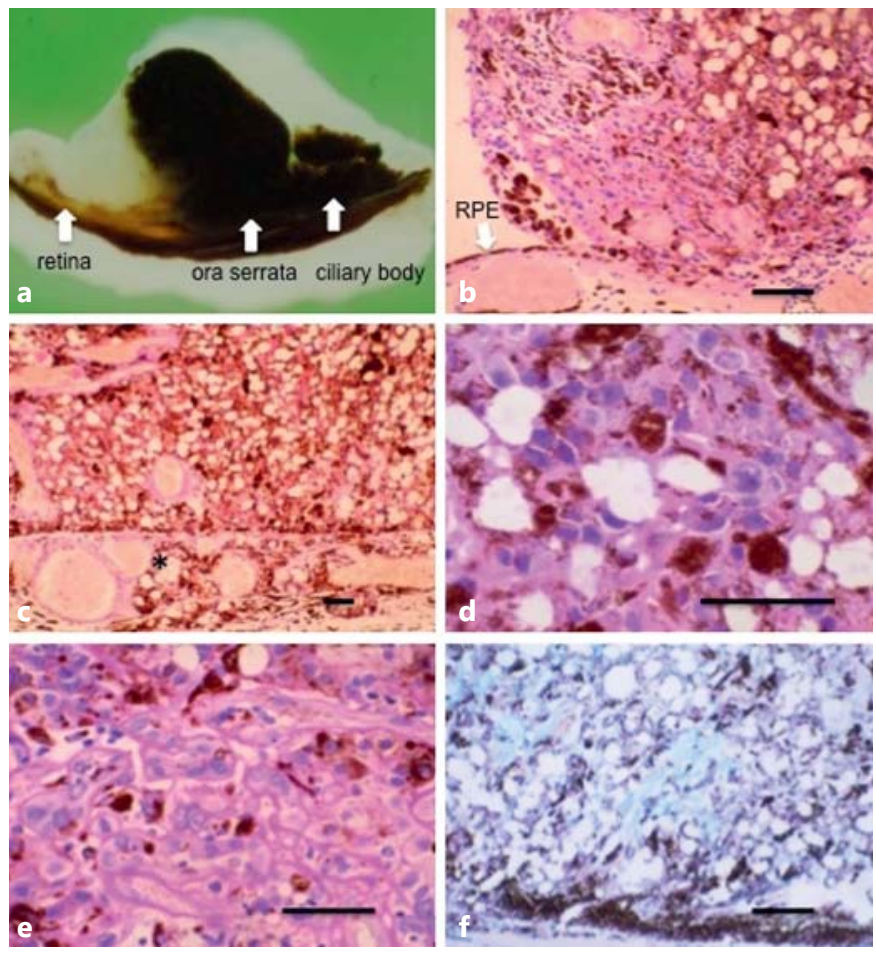

Fig. 2. Gross and light microscopic findings of the tumor. a Gross appearance of the lesion showing a darkly pigmented mass arising from the ora serrata. The tumor is $7.5 \times 5.5 \mathrm{~mm}$ in basal diameter and $3.5 \mathrm{~mm}$ in thickness. The left side is near the retina, and the right side is near the ciliary body (white arrows). b The tumor showing abrupt elevation from the retinal pigment epithelium (RPE). H\&E staining; bar $=100 \mu \mathrm{m}$. c A part of tumor cells invading the choroid (asterisk). There are no nevoid cells in the choroid. $\mathrm{H} \& \mathrm{E}$ staining; bar $=100 \mu \mathrm{m}$. d Many vacuoles and heavily pigmented granules are seen throughout the tumor. Atypical nuclei with prominent nucleoli are evident in tumor cells. H\&E staining; bar $=50 \mu \mathrm{m}$. e The basement membrane showing a positive reaction to periodic acid-Schiff staining, and tubular or lobular proliferation are seen; bar $=50 \mu \mathrm{m}$. $\mathbf{f}$ Intracytoplasmic vacuoles showing a positive reaction to colloidal iron staining; bar $=100 \mu \mathrm{m}$.

RPE (Fig. 2b). The main part of the tumor was present on Bruch's membrane and focally invaded the retina at its apex and the choroid at its base. There were no nevoid cells in the choroid, which is consistent with the typical appearance of malignant melanoma of the choroid (Fig. 2c). Most of the tumor cells contained a number of intracytoplasmic vacuoles and were heavily pigmented. The tumor cells had a nuclear pleomorphism and prominent nucleoli (Fig. 2d). Periodic acid-Schiff staining revealed apparent basement membrane around the tubularly proliferated tumor cells (Fig. 2e). A small part of the tumor showed a tubular or lobular proliferation pattern of the cells with relatively few melanin granules (Fig. 2e). Intracytoplasmic vacuoles showed partial positivity for colloidal iron staining at the ciliary body (Fig. 2f).

Immunohistochemical staining of the tumor cells were positive for S-100 protein (i.e., calcium-binding protein), HMB-45 (i.e., a melanoma-specific antigen), epithelial membrane antigen (EMA), and AE-1 (i.e., a low-molecular-weight cytokeratin marker) (Fig. 3).

The appearance of the tumor cells on electron microscopy exhibited intracytoplasmic vacuoles that contained an amorphous substance with a high electron density. There were cell membrane structures among the vacuoles (Fig. 4a). The tumor cells contained many melanin granules and showed pleomorphic nuclei and prominent nucleoli. The cytoplasm of the tumor cells, comprising foamy cells, contained many developmental rough endoplasmic reticulum areas and mitochondria (Fig. 4b). Most of the melanosomes were mature and round (Fig. 4b). We confirmed the presence of tight junctions between the tumor cells, which indicated that this tumor was derived from the RPE (Fig. 4c, d). From the above findings, our final histopathological diagnosis was a RPE adenocarcinoma.

\section{Discussion}

Because there are only a few reports on RPE adenocarcinomas, it is very difficult to distinguish between RPE adenocarcinomas and malignant melanomas of the choroid [1-3]. Currently, RPE adenocarcinomas are treated as malignant melanomas of the choroid without histological confirmation [6]. Indeed, our initial clinical diagnosis was malignant melanoma of the choroid [5].

Neoplasms of the RPE are uncommon. A RPE adenocarcinoma readily has been caused by reactive hyperplasia and metaplasia following trauma, inflammation, or degenerative processes that involve the choroid or retina. These may also occur without an apparent cause [7-9]. In the case reported herein, there was no history of any of these events.

A particularly important feature of a RPE adenocarcinoma is the occasional presence of a dilated retinal feeder vessel, a feature very rarely found with malignant melanoma of the choroid. Shields et al. [4] found dilated retinal vessels in 8 out of $13 \mathrm{RPE}$ tumors. Dilated retinal vessels are observed rarely when malignant melanoma of the choroid invades the retina. The blood supply of a uveal melanoma arises from the rich choroidal vasculature, whereas RPE tumors are supplied by the retinal vasculature. Fluorescence angiography confirmed that retinal vessels supplied the tumor in our case. RPE adenocarcinoma classically appears as a heavily pigmented fundus tumor, occasionally with surrounding subretinal fluid and exudative retinopathy with yellowish deposits. The fundus lesion in our patient resembled the RPE tumors reported previously by Shields et al. [10-12]. These features are rare in malignant melanomas of the choroid.
Ocul Oncol Pathol 2018;4:38-43 DOI: $10.1159 / 000477735$
Mori/Takahashi 

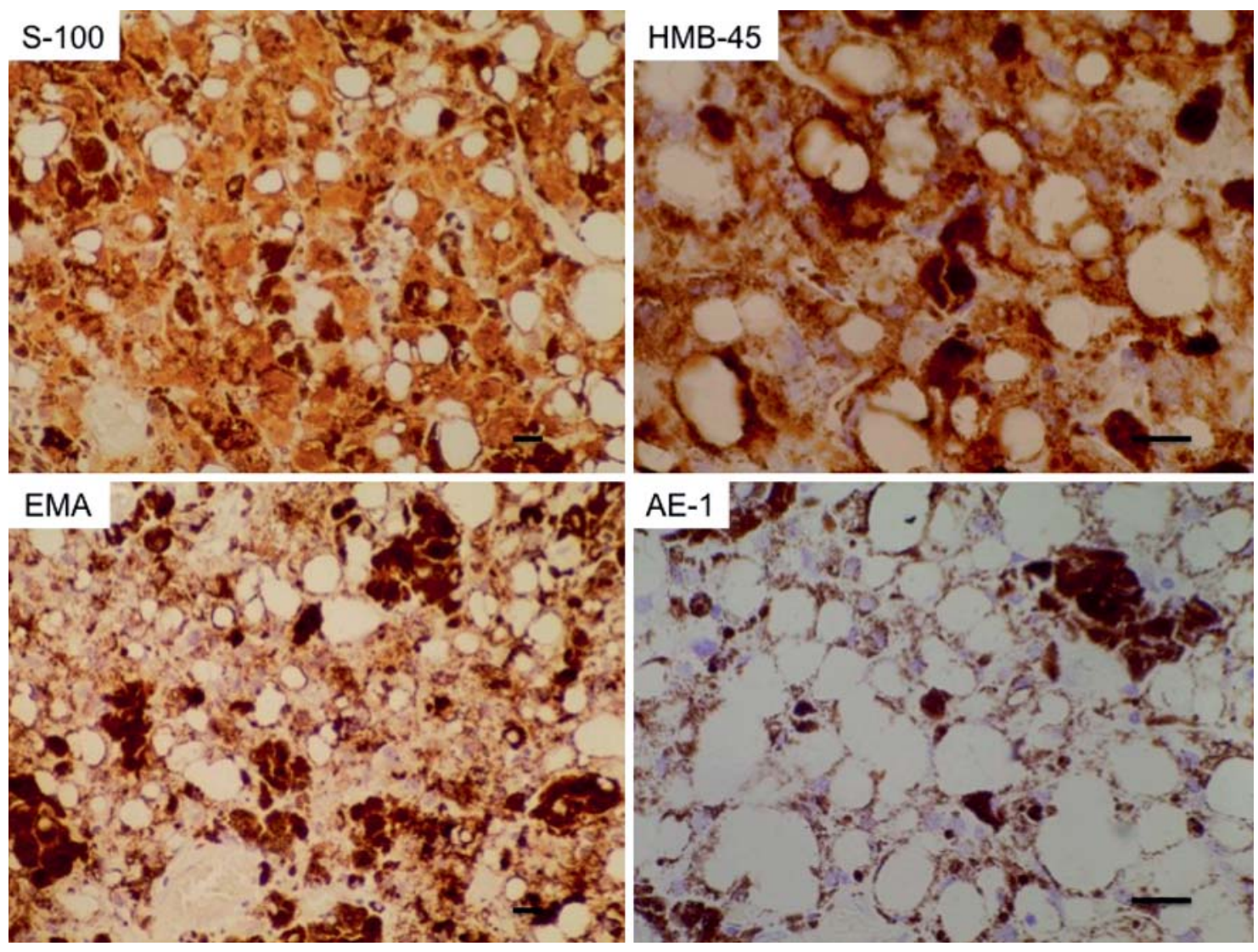

Fig. 3. Immunohistochemical findings. Tumor cells showing positive immunoreactivitiy to melanocytic markers (S-100 and HMB-45) and epithelial markers (EMA and AE-1); bar $=100 \mu \mathrm{m}$.

Biopsy can be used to distinguish RPE adenocarcinoma from malignant melanoma of the choroid, but this method is very difficult, even for experienced pathologists $[4,12]$. Most RPE tumors require treatment because RPE tumors show progressive growth that causes visual loss and destroys the eye structure $[4,12]$. Radiation therapy is one treatment option but does not appear to be highly effective $[4,13]$. There has been rare documentation of extraocular extension or metastasis [9]. We therefore performed therapeutic diagnosis by tumor resection. With regard to pathological findings, it was difficult to distinguish between RPE adenocarcinoma and malignant melanoma of the choroid. In fact, a pathologist at our hospital initially diagnosed this case as a malignant melanoma of the choroid [5]. In addition, it is difficult to distinguish RPE adenocarcinoma from RPE adenoma. The reasons for finally making the diagnosis of RPE adenocarcinoma in our case were the following. There were no nevoid cells in the choroid, which should indicate malig-

Pathologic Findings of RPE

Adenocarcinoma nant melanoma. The main part of the tumor protruded into the vitreous cavity from the RPE. Only a small part of the tumor showed invasion to the choroid. Tumor cells showed nuclear pleomorphism, which indicated RPE adenocarcinoma, not adenoma.

Histopathologically, RPE tumors were classified into vacuolated, tubular, and mixed types by Font RL [14]. We could confirm both vacuolated and tubular appearances in our case, which meant mixed type.

The immunohistochemical evidence supporting a RPE origin was unequivocal [1]. Shields et al. [15] reported that RPE tumor cells showed coexpression for epithelial markers and melanocytic markers. In our case, we obtained positive staining for both markers.

In published retrospective case series, very few reports showed electron microscopic findings of RPE tumors [1, 16]. This case is the first to report electron microscopic findings of an RPE adenocarcinoma in an Asian patient. Electron microscopy showed tight junctions between tu-

Ocul Oncol Pathol 2018;4:38-43 DOI: $10.1159 / 000477735$ 

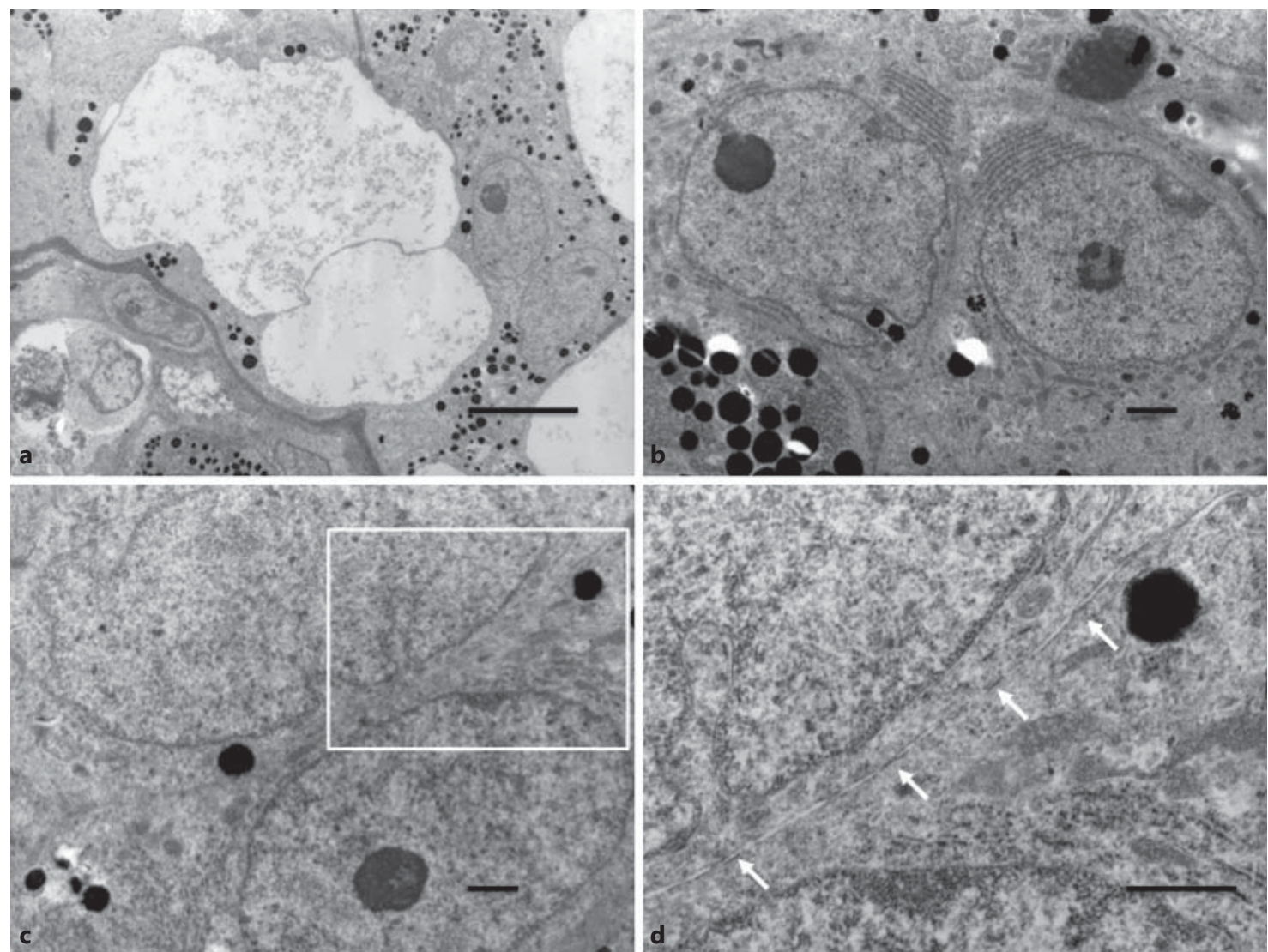

Fig. 4. Electron microscopic findings. a Tumor cells containing intracytoplasmic vacuoles with amorphous materials; bar $=10 \mu \mathrm{m}$. b Tumor cells containing round melanin granules and abundant rough endoplasmic reticulum and mitochondria in the cytoplasm. The nucleus showing prominent nucleoli; bar $=1 \mu \mathrm{m}$. c Lower magnification of the intercellular junctions. $\mathbf{d}$ Higher magnification image of the inset in $\mathbf{c} ; \mathrm{bar}=1 \mu \mathrm{m}$. $\mathbf{d}$ Intercellular junctions are observed between the tumor cells (white arrows); bar $=1 \mu \mathrm{m}$.

mor cells; an additional finding that was consistent with RPE origin.

Although 3 deaths from metastasis of RPE tumors were reported by Garner [9], our patient continued to do well with no evidence of metastatic disease or orbital recurrence.

Although malignant melanoma of the choroid is the most frequent malignant intraocular tumor, it is important to consider other malignancies including adenocarcinoma of the RPE. Immunohistochemical and electron microscopic examinations are valuable for precise diagnosis.

\section{Statement of Ethics}

Written informed consent was obtained from the patient for the publication of this case report and the accompanying images.

\section{Disclosure Statement}

The authors declare that there are no financial or other conflicts of interest to disclose.

References

1 Karin U, Loeffler KU, Kivela T, Borgmann H, Witschel $\mathrm{H}$ : Malignant tumor of the retinal pigment epithelium with extraocular extension in a phthisical eye. Graefes Arch Clin Exp Opthalmol 1996;234:70-75.

2 Melis P, Shields CL, Marr BP, Eagle RC Jr, Shields JA: Retinal pigment epithelial tumor in a young Asian female. Euro Ophthalmol 2009;3:487-489.

3 Shields JA, Eagle RC Jr, Barr CC, Shields CL, Jones DE: Adenocarcinoma of the retinal pigment epithelium arising from a juxtapapillary histoplasmosis scar. Arch Ophthalmol 1994; 112:650-653. 
4 Shields JA, Shields CL, Gunduz K, Eagle RC Jr: Neoplasms of the retinal pigment epithelium: the 1998 Albert Ruedemann, Sr, memorial lecture, part 2. Arch Ophthalmol 1999; 117:601-608.

5 Taomoto M, Nishimura T, Takahashi K, Miyazaki T, Uyama M, Uchida K: A case of choroidal malignant melanoma treated by transcleral resection (in Japanese). Rinsho Ganka 1999;53:1927-1932.

6 Sommacal A, Campbell RJ, Helbig H: Adenocarcinoma of the retinal pigment epithelium. Arch Ophthalmol 2003;121:1481-1483.

7 Shields JA, Shields CL: Intraocular Tumors. A Text and Atlas. Philadelphia, WB Saunders, 1992, pp 437-460.

8 Green WR: Retina; in Spencer WH, Bilyk JR, Eagle RC Jr, et al. (eds): Ophthalmic Pathology: An Atlas and Textbook. Philadelphia, WB Saunders, 1996, vol 2, pp 1312-1316.
9 Garner A: Tumors of the retinal pigment epithelium. Br J Ophthalmol 1970;54:715-723.

10 Shields CL, Mashayekhi A, Ho T, Cater J, Shields JA: Solitary congenital hypertrophy of the retinal pigment epithelium: clinical features and frequency of enlargement in $330 \mathrm{pa}$ tients. Ophthalmology 2003;110:1968-1976.

11 Shields JA, Shields CL, Singh AD: Acquired tumors arising from congenital hypertrophy of the retinal pigment epithelium. Arch Ophthalmol 2000;118:637-641.

12 Shields JA, Shields CL: Tumors and related lesions of the pigment epithelium; in Shields JA Shields CL (eds): Intraocular Tumors. An Atlas and Textbook, ed 2. Philadelphia, Lippincott Williams \& Wilkins, 2008, pp 432-483.
13 Shields JA, Shields CL, Eagle RC Jr, Singh AD: Adenocarcinoma arising from congenital hypertrophy of the retinal pigment epithelium. Arch Ophthalmol 2001;119:597-602.

14 Font RL, Zimmerman LE, Fine BS: Adenoma of the retinal pigment epithelium: histochemical and electron microscopic observations. Am J Ophthalmol 1972;73:544-554.

15 Shields JA, Eagle RC Jr, Dutton J, Ehya H, Shields CL: Adenocarcinoma of the retinal pigment epithelium clinicopathologic correlation with paradoxical immunohistochemical findings. JAMA Ophthalmol 2014;132: 1249-1252.

16 Don Minckler, Allen AW Jr: Adenocarcinoma of the retinal pigment epithelium. Arch Ophthalmol 1978;96:2252-2255. 